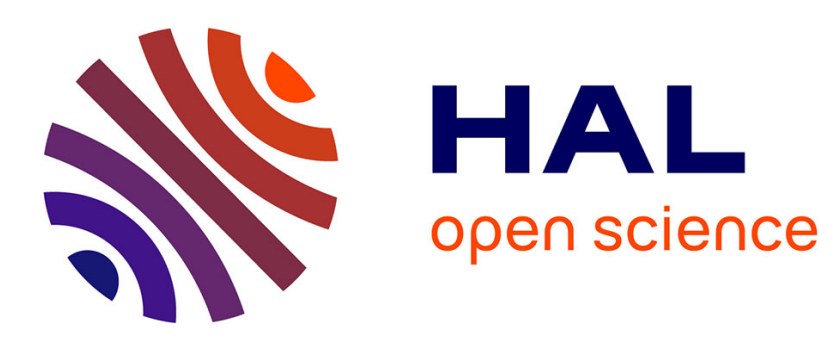

\title{
Improved curvature-based registration methods for high-precision dimensional metrology
}

Rindra Rantoson, Hichem Nouira, Nabil Anwer, Charyar Mehdi-Souzani

\section{To cite this version:}

Rindra Rantoson, Hichem Nouira, Nabil Anwer, Charyar Mehdi-Souzani. Improved curvaturebased registration methods for high-precision dimensional metrology. Precision Engineering, 2016, 10.1016/j.precisioneng.2016.05.002 . hal-01363750

\section{HAL Id: hal-01363750 \\ https://hal.science/hal-01363750}

Submitted on 11 Sep 2016

HAL is a multi-disciplinary open access archive for the deposit and dissemination of scientific research documents, whether they are published or not. The documents may come from teaching and research institutions in France or abroad, or from public or private research centers.
L'archive ouverte pluridisciplinaire HAL, est destinée au dépôt et à la diffusion de documents scientifiques de niveau recherche, publiés ou non, émanant des établissements d'enseignement et de recherche français ou étrangers, des laboratoires publics ou privés. 


\title{
Improved curvature-based registration methods for high- precision dimensional metrology
}

\author{
Rindra RANTOSON ${ }^{1}$, Hichem NOUIRA ${ }^{1}$, \\ Nabil ANWER ${ }^{2}$ and Charyar MEHDI-SOUZANI ${ }^{2}$
}

(1): Laboratoire Commun de Métrologie (LNE-CNAM), Laboratoire National de Métrologie et d'Essais (LNE), 1 rue Gaston Boissier, 75015 Paris, France.

(2): Laboratoire Universitaire de Recherche en Production Automatisée (LURPA), Ecole Normale Supérieure de Cachan, Université Paris Sud, 61 avenue du Président Wilson, 94235 Cachan Cedex, France.

* Corresponding authors: hichem.nouira@,1ne.fr \& rindra.sanders@yahoo.fr

Keywords: 3D Registration; Discrete Curvature; Ransac Transformation; Hough Transformation; Coarse registration; ICP variant; Computed tomography.

Abstract: Multiple measurements using various data acquisition systems are generally required to substancially enhance measurement accuracy, reliability and holisticity of freeform shapes. The obtained multiple measurement data of the shape are transformed and fused into a common coordinate system within a registration technique involving coarse and fine alignments. Standardized methods have been established for fine registration such as Iterative Closest Points (ICP) and its variants. For coarse registration, no conventional method has been adopted yet despite a significant number of techniques which have been developed in the literature to supply an automatic rough matching between data sets.

The work presented in this paper proposes an improvement of registration techniques by the consideration of new discrete curvature parameters. Two main issues are addressed in this paper: the coarse registration and the fine registration. For coarse registration, two novel automated methods based on the exploitation of discrete curvatures are presented: an enhanced Hough Transformation (HT) and an improved Ransac Transformation. The use of curvature features in both methods aims to reduce computational cost. For fine registration, a new variant of ICP method is proposed in order to reduce registration error using curvature parameters. A specific distance considering the curvature similarity is combined with Euclidean distance to define the distance criterion used for correspondences searching. Additionally, the objective function is improved by combining the point-to-point (P-P) minimization and the point-to-plane (P-Pl) minimization with automatic weights. The algorithms are applied on simulated and real data performed by a computed tomography (CT) system. The obtained results reveal the benefit of the proposed improved curvature-based registration methods. 


\section{Introduction}

Several measurements by means of different optical, capacitive, tomography and tactile probing systems are commonly required for the assessment of the shape of freeform workpieces while controlling reliability and accuracy of the results. Data obtained from one or several measuring machines equipped with one or several probing systems are characterized by different coordinate systems, different measurement uncertainties, different and limited overlapping sections and different spatial organization of points. These data are then aligned and fused into a common coordinate system using a registration technique [1-2]. During the registration process the optimal transformation parameters between two data sets (scene data and model data) endowed with overlapping sections is computed through two phases: coarse and fine registrations.

The coarse registration roughly aligns the two data sets with a lower resolution from a global view. The outcome alignment is thereafter optimized through the fine registration for a higher resolution. The coarse registration result is important since the accuracy of the fine registration depends on it. Additionally, the search for correspondences in the overlapping sections of both data sets for the transformation estimation constitutes a crucial step. Numerous coarse registration attempts are proposed to deal with or without this matching step while for fine registration the closest point criterion is standardly used.

For coarse registration, although the matching step can be achieved manually by selecting the identified natural or artificial corresponding marker points, miscellaneous automatic coarse registration techniques are currently proposed in the literature [3]. Among them, some seek to avoid the corresponding step using Principal Component Analysis (PCA) [4] or maximum likelihood techniques [5] while for the other approaches, matching step represents an important part of the algorithm, e.g. feature-based methods and segmentation [6]. Feature-based methods have been studied to a large extent $[7,8]$. The characteristics used to find correspondences can be curvatures, signature of salient features, graph model, color and texture information, normal vectors, intrinsic invariants, geometric features using second order local surface approximations, integral descriptor and many other techniques $[9,10]$. Furthermore, a variety of surface descriptors have been used for computing feature point signatures for an automatic approximate alignment of partially overlapping surfaces [11,12]. Feature-based methods are generally sensitive to noise. Thus, a denoising or smoothing is necessary prior to the application of the method.

Furthermore, registration using PCA is fast and reliable when data sets are endowed with large similarity and no symmetries [13].HT [13,14] and Ransac [15] cope with PCA limitations while dealing also with smooth or textureless objects. Nevertheless, the exhaustive search in HT presents 
runtime weakness and high memory consumption while Ransac computational time can be heavily affected by a significant volume of data.

Iterative Closest Point (ICP) $[16,17]$ has been established to optimize the rough alignment issue of coarse registration. Several ICP variants, affecting all phases of the algorithm from matching of points and selection of correspondences to the minimization strategy, have been carried out to improve the algorithm performance in term of speed, range and rate of convergence and robustness [18]. Corresponding search is a crucial step of registration while it considerably affects the results accuracy and conditions the range and the rate convergence. Some ICP variants have been developed for speeding up the convergence using various data structure like k-D tree [19], spatial bins and multiple Z-Buffer techniques [20], etc. Numerous attempts have also been made to robustify ICP regarding the obtained matched points used in the minimization problem formulation [21,22]. Instead of using all matched points, different sampling strategies have been adopted to compute the transformation using only some selected point pairs while attempting to overcome local minima [18]. Wrong pairs are rejected using robust statistics such as Least Median of Squares (LMedS) [23], Least Trimmed Squared (LTS) [24], etc. Euclidean distances are commonly used to evaluate the similarity between correspondences such as P-P (point-to-point) distance [16] and P-P1 (point-to-plane) distance [23]. Regarding the algorithms for solving the minimization problem, Unit Quaternion (UQ) method was used in the original version of ICP [16]. Nevertheless, Singular Value Decomposition (SVD) has been shown to yield the best global accuracy and stability [25].Different minimization strategy and error metric have been explored. Although P-Pl minimization method has been proved to be robust to noises, inaccuracies can be induced if neighbor points present high curvatures due to the least squares estimation of the tangent plane.

Considering all of the above studies, the purpose of the presented work is twofold: Firstly, a novel registration framework is proposed for coarse registration to allow a considerable reduction of Hough and Ransac cost. These approaches are combined with the exploitation of discrete curvature features. The curvature parameters including the principal curvatures, the principal directions, the curvedness, the shape index and its corresponding surface type are preliminary computed using a discrete curvature calculation method. For HT method, the local transformation parameters are computed only for vertices of the same surface type, which considerably reduces the time processing and the required memory storage in Hough table. For Ransac method, a combination of geometric distance with curvature features (surface type and curvedness) constraints is proposed to improve the registration error and to accelerate convergence. Secondly, a curvature-based fine registration method is proposed. The developed method combines P-Pl registration with P-P minimization with an automatic weighting. Weights are attributed according to the curvature 
features preliminary calculated at each point. Thus, according to the obtained surface type point, PPl will be more or less privileged in the objective function formulation. Furthermore, curvature feature distance has been established for correspondences searching defined from the principal curvatures and combined with Euclidean distance for reliability.

The rest of the paper is organized as follows: In Section 2, a brief presentation of discrete curvatures estimation methods and curvature measures are performed, along with a detailed description of a tensor-based method. In Section 3 and Section 4, curvature-based Hough Transformation and curvature-based Ransac are presented. In Section 5, the new curvature-based ICP variant is developed. Experimental results based on X-ray micro-Computed Tomography measurements and quantitative simulation results are illustrated and discussed in Section 6. In Section 7 the main aspects of the developed methods are discussed, as well as future research directions.

\section{Curvatures features extraction}

\section{1- Discrete curvature estimation methods}

Most of the classical discrete curvature estimation methods are based on the polygon mesh surfaces. However, due to the piecewise smoothness of the input mesh, the discrete curvature estimation is subject to various definitions [26,27]. According to Meek and Walton [28], the classical methods of discrete estimation based on polygon mesh surfaces could be classified into three basic categories: one may approximate a local quadric surface at a given vertex mesh and then apply the derivatives to obtain the curvatures. One may discretize the mathematic formulae that give the curvature information of continuous surface and extend the notations to discrete domains [27]. One may use the tensor based techniques for discrete curvature estimation [26]. This approach is adopted here.

\section{2- Curvature definition}

Assuming $\Sigma$ an orientable surface embedded in the three dimensional Euclidean space $\mathbb{R}^{3}$ and $\vec{n}(p)$ the surface normal at a given point $p \in \Sigma$, curvatures are defined to measure the local bending of $\Sigma$. Take the given point $p$, for each unit direction $\vec{u}$ on its tangent plane $T_{\Sigma}(p)$, the normal curvature $\kappa_{n}(\vec{u})$ is defined as the curvature of the curve that belongs to both the surface itself and a perpendicular plane containing both $\vec{n}(p)$ and $\vec{u}$ (Fig.1(a)). Formally, the normal curvature is defined in Eq. 1.

$$
\kappa_{n}(\vec{u})=S_{p}(\vec{u}) \cdot \vec{u}
$$

Where, $S_{p}(\vec{u})$ denotes the shape operator at point $p$ along the direction $\vec{u}$ and it is defined as the derivate of $\vec{n}(p)$ with the tangent direction $\vec{u}$ (Eq.2).

$$
S_{p}(\vec{u})=-\nabla_{\vec{u}} \vec{n}(p)
$$


$\nabla_{\vec{u}} \vec{n}(p)$ indicates the gradients of $\vec{n}(p)$ along $\vec{u}$ at point $p$.

The shape operator can be shown to be symmetric. Its eigenvalues and the corresponding eigenvectors are respectively called the principal curvatures and the principal directions denoted $\vec{T}_{1}$ and $\vec{T}_{2}$ (Fig.1(a)). Both the principal curvatures and the principal directions can be recovered from the shape operator matrix.

\section{3- Cohen-Steiner and Morvan method}

Cohen-Steiner and Morvan [26] proposed a method to estimate the discrete curvature tensor on polygon mesh by elaborating a curvature measure regarded as a discrete shape operator matrix inspired from the smooth case. For that purpose, a curvature tensor is first estimated at each vertex. Thereafter, in order to build the continuous curvature tensor field over the whole surface, the piecewise linear curvature tensor field is built by interpolating these values across triangles. Since it is not a natural way to evaluate the discrete curvatures at an isolated vertex, one should consider the integrals of curvatures over a given local region around a vertex. The integral of curvatures associated with local region is called curvature measure and its anisotropic representation is provided in Eq.3 for a local region $B$ around a vertex $p$ on a given polyhedral mesh $\Sigma$ (Fig.1(b)).

$$
\begin{gathered}
\bar{H}_{V}(B)=\sum_{e \in E} \frac{\operatorname{length}(e \cap B)}{2}\left[(\beta(e)-\sin \beta(e))\left(\vec{e}^{+} \times \vec{e}^{+T}\right)\right. \\
\left.\left.+(\beta(e)+\sin \beta(e))\left(\vec{e}^{-} \times \vec{e}^{-T}\right)\right]\right]
\end{gathered}
$$

Where $E$ is the collection of all the mesh edges in $B \cdot \vec{e}^{+}$and $\vec{e}^{-}$denote the normalized sum and difference of unit normal vectors of the triangles incident at the edge $e$ respectively. length $(e \cap B)$ is the length of the edge $e$ in the local region $B . \beta(e)$ is the dihedral angle between the two normal vectors of the triangle incident with the edge $e$ (Fig.1(c)).

The shape operator $\bar{H}_{V}(B)$ is a $3 \times 3$ symmetric matrix which has three eigenvalues and three eigenvectors. The three eigenvectors correspond to the maximum principal direction, the minimum principal direction and the normal vector at the given vertex respectively. Therefore, the first two maxima eigenvalues of $\bar{H}_{V}(B)$ are the two principal curvatures at the given vertex. A simpler form of $\bar{H}_{V}(B)$ denoted $\overline{\widetilde{H}}_{V}(B)$ which has the same eigen vectors as $\bar{H}_{V}(B)$ but swapped eigenvalues has been supplied by Zhao et al. [13] (Eq.4) and adopted in our implementation for computation convenience.

$$
\overline{\widetilde{H}}_{V}(B)=\frac{1}{A} \sum_{\mathrm{e} \in \mathrm{E}} \beta(\mathrm{e}) . \text { length }(e \cap B) \cdot\left(\vec{e} \times \vec{e}^{T}\right)
$$

Where $A$ is the area of the considered local region $B$ and $\vec{e}$ is a unit vector of the edge $e$. 
Our considered local region $B$ is defined by the Voronoi-based cell generated on the one-ring neighborhood of a vertex $p$ (Fig.1(b)) and an additional coefficient $\lambda_{e}$ has been integrated in the formulation of $\overline{\widetilde{H}}_{V}(B)$ (Eqs.5-10) to improve results quality.

$$
\overline{\widetilde{H}}_{V}(B)=\frac{1}{A} \sum_{e \in E} \lambda_{e} \cdot \beta(e) . \text { length }(e \cap B) \cdot\left(\vec{e} \times \vec{e}^{T}\right)
$$

Where,

$$
\begin{aligned}
& \lambda_{e}=\frac{\cos ^{-1}(\overrightarrow{n(p),}, \overrightarrow{n(e)})}{\sum_{\mathrm{e} \in \mathrm{E}} \cos ^{-1}(\overrightarrow{n(p)}, \overrightarrow{n(e))}} \\
& \vec{n}(e)=\frac{\vec{n}_{1}+\vec{n}_{2}}{\left\|\vec{n}_{1}+\vec{n}_{2}\right\|} \\
& \vec{n}(p)=\frac{\sum_{\mathrm{i}=1}^{\varkappa} \omega_{i} \cdot \vec{n}_{i}}{\left\|\sum_{\mathrm{i}=1}^{\mathrm{N}} \omega_{i} \cdot \vec{n}_{i}\right\|} \\
& \vec{n}_{i}=\frac{\vec{e}_{i 1} \times \vec{e}_{i 2}+\vec{e}_{i 2} \times \vec{e}_{i 3}+\vec{e}_{i 3} \times \vec{e}_{i 1}}{\left\|\vec{e}_{i 1} \times \vec{e}_{i 2}+\vec{e}_{i 2} \times \vec{e}_{i 3}+\vec{e}_{i 3} \times \vec{e}_{i 1}\right\|} \\
& \omega_{i}=\frac{A_{i} / d_{i}^{2}}{\sum_{i=1}^{\varkappa} A_{i} / d_{i}^{2}}
\end{aligned}
$$

$\vec{e}_{i 1}, \vec{e}_{i 2}$ and $\vec{e}_{i 3}$ are the vector units of a given face $i$ in the meshing. $d_{i}$ is the distance between the vertex $p_{i}$ and the centroid of the face $i$ (Fig.1(d)).

\section{4- Shape index and curvedness calculation}

The main indicators for surface type recognition utilized here are the shape index and the curvedness. Both specify the second order geometry of a shape. The shape index is a quantitative measure of the local surface type of a point on a surface. It as a single value within the range $[-1,1]$ and mathematically defined in Eq.11.

$$
s=-\frac{2}{\pi} \tan ^{-1}\left(\frac{\kappa_{1}+\kappa_{2}}{\kappa_{1}-\kappa_{2}}\right),\left(\kappa_{1} \geq \kappa_{2}\right)
$$

Curvedness, as a complementary parameter to the shape index, is a bending energy represented by a positive number that specifies the amount or intensity of the surface curvatures (Eq.12).

$$
c=\sqrt{\frac{\kappa_{1}^{2}+\kappa_{2}^{2}}{2}}
$$

Where, $\kappa_{1}$ and $\kappa_{2}$ are respectively the maximum and minimum principal curvatures of the local surface.

\section{5- Surface type classification}


From the shape index, nine basic surface types in a continuous way defined in [29] are assumed here. This definition is more convenient than the classical one based on Gaussian and Mean curvature. However, since planar shapes were not defined in [29], a surface type for planar shape has been established when shape index values are equal to 2 (Table I). A unique integer called surface type label $L$ is assigned to each of the ten surface types for convenience of the classifying and processing (Table I) [13].

\section{6- Algorithm}

The algorithm for curvature features extraction can be summarized for each vertex of a considered data as follows:

- Step 1: Calculation of the shape operator matrix $\overline{\widetilde{H}}_{\mathrm{V}}(\mathrm{B})$;

- Step 2: Eigen value decomposition of $\overline{\widetilde{\mathrm{H}}}_{\mathrm{V}}(\mathrm{B})$;

- Step 3: Calculation of the shape index and the curvedness;

- Step 4: Calculation of the surface type.

\section{Enhanced Hough Transformation (HT)}

\section{1- Classical HT}

HT avoids the priori knowledge of correspondences using an exhaustive search to estimate the rough transformation between the scene data and the model data. For that purpose, local frames are first calculated at each point for both data sets. Thereafter, each frame of the scene data has to be paired with each one from the model data providing $m \times n$ transformations if $m$ and $n$ are respectively the number of points in scene data and in model data. All resulted new transformations are stored in Hough table while their respective frequencies of occurrence are updated by using an incrementing counter for each stored transformation (Fig.2(a)). Therefore, the most frequent transformation in Hough table is retained for rough alignment based on the following assumption. Transformations calculated from correct point correspondences result in the same transformations, whereas all other transformations are distributed more or less randomly in the Parameter Space (PS). Hence, a peak in Hough table is expected at the position of the searched transformation.

\section{2- Proposed Curvature-based HT}

Curvature features are combined with HT algorithm to reduce the number of Hough operations while optimizing the memory consumption required for Hough table, which affects consequently the computational time. Transformation parameters are computed only for points of the same surface type (Fig.2(b)). Thus, the basic transformation $o(m \times n)$ operations are considerably reduced according to the number of the identified surface types in both data sets and 
the partition of points in the generated classes. For example, if 10 surface types are identified for both data sets which points are uniformly classified in these 10 surface type classes, the corresponding transformation operation is then given by $\mathrm{o}\left(10 \times\left[\frac{\mathrm{n}}{10} \times \frac{\mathrm{m}}{10}\right]\right)=\mathrm{o}(\mathrm{m} \times n / 10)$.

Furthermore, additional and optional stopping criteria were defined to improve the algorithm performance. Some heuristic could be added to speed up the algorithm. Here, the algorithm stops if the maximum number of the counter is greater than a threshold $T_{h}$ multiplied by the second maximum value of the counter at a certain iteration of the loop (Eq.13).

$$
\max \{\text { counter }\}>T_{h} . \max \{\text { counter } \backslash\{\max (\text { counter })\}\}
$$

This criterion can be strengthened if a prior knowledge of the overlapping sections is available. Moreover, the transformation operation can be further decreased by applying the transformation parameters calculation only on points of one or some specific surface types. Indeed, since a priori knowledge of the surface types of points in the overlapping sections is available, transformations parameters can be calculated only from these points. For example, if the overlapping sections contain some planes (which class is denoted respectively $P^{\prime}$ and $Q^{\prime}$ in scene and in model data), when considering points classified as planes for the transformation calculation, the transformation operation will considerably decrease and will be no more than $\mathrm{o}\left(m^{\prime} \times n^{\prime}\right) . m^{\prime}$ and $n^{\prime}$ are respectively the number of points which surface type is plane in scene data and in model data (i.e. in $P^{\prime}$ and $Q^{\prime}$ ).

\section{3- Algorithm}

The proposed algorithm can be summarized as follows:

- Step 1: Compute local frames $V_{i}$ and $V_{j}$, which can be the Eigen vectors resulted from Eigen value decomposition of the shape operator matrix $\widetilde{\widetilde{H}}_{V}(B)$;

- Step 2: Initialization of Hough table and the counter;

- $\quad$ Step 3: Compute transformation from $V_{i}$ and $V_{j}$ in $P^{\prime} \& Q^{\prime}$ (Eq.14);

$$
R_{i \rightarrow j}=V_{j} V_{i}^{T}, T_{i \rightarrow j}=p_{j}-R_{i \rightarrow j} p_{i}
$$

- Step 4: Test if $\left(R_{i \rightarrow j} T_{i \rightarrow j}\right)$ belongs to Hough table (Ht) (Eq.15);

$$
\left(R_{\text {opt }}, T_{\text {opt }}\right)=\arg \min _{R, T \in H t}\left[\operatorname{dist}\left(\left(R_{i \rightarrow j}, T_{i \rightarrow j}\right)-(R, T)_{H t}\right)<\varepsilon\right]
$$

- Step 5: Update the counter or Hough table;

- Step 6: Extract the retained transformation by selecting the most frequent transformation in Hough table;

If $N=m \times n$ is the number of operations in the initial HT algorithm with $\mathrm{m}$ and $\mathrm{n}$ the number of points respectively in scene data and in model data, $N$ is equal to $\sum_{\mathrm{L}=1}^{\mathrm{K}}\left(m^{\prime} \times n^{\prime}\right)_{\mathrm{L}}$ for the enhanced 
algorithm. Where $K$ is the number of the selected surface types to be considered within their respective number of points $m^{\prime}$ and $n^{\prime}$ identified in scene data and in model data.

\section{Improved Ransac}

\section{1- Ransac-based darces registration}

The Ransac-based darces algorithm is addressed as follows:

- Step 1: Initialization: three points $\left(p_{1}, p_{2}, p_{3}\right)$ are randomly picked in scene data $P$.

- Step 2: Searching of their respective corresponding candidates' points $\left(q_{1}, q_{2}, q_{3}\right)$ in model data $Q$ using distance constraints.

- Step 3: Calculation of $(R, T)$ from 1 candidate sets of 3 point pairs (Eq.16).

$$
(R, T)=\min _{R, T} \sum_{i=1}^{N_{\Omega}=3}\left\|R p_{i}+T-q_{i}\right\|^{2}
$$

Steps 1,2 and 3 are repeated $\mathrm{k}$ times providing then $k^{\prime}=\sum_{b=1}^{k} l_{b}$ transformations: $\left(R_{1}, T_{1}\right)$, $\left(R_{2}, T_{2}\right) \ldots\left(R_{k^{\prime}}, T_{k^{\prime}}\right)$.

- Step 4: Calculation of the transformed points ${P^{\prime}}_{1}, P^{\prime}{ }_{2} \ldots P^{\prime}{ }_{k \prime}$ by applying respectively the $k^{\prime}$ above transformations.

$$
P^{\prime}=R P+T
$$

- Step 5: Calculation of the number $n_{k^{\prime}}$ of closest points between each $P_{i}^{\prime}$ and $Q, i=$ $1 \ldots k^{\prime}$.

- Step 6: Identification and selection of the transformation with the maximum value of $n$.

Distance constraints related to Step 2 are used for finding $q_{2}$ and $q_{3}$ :

- $q_{2}$ candidates are points on the sphere centered at $q_{1}$ and which ray is the distance between $p_{1}$ and $p_{2}$, Fig.3(a).

- $q_{3}$ candidates are points on the circle centered at $h q_{12}$ and which ray is the distance between $h p_{12}$ and $p_{3}$ (Fig.3(b)).

\section{2- Curvature-based Ransac registration}

The identical principle for HT enhancements approach has been integrated in Ransac algorithm to improve its performance using an additional curvature parameter. Distance constraints are combined with curvature feature constraints (surface type and curvedness) which are applied first in the matching step to reduce the corresponding points searching area to $\psi\left(p_{i}\right)$ (Eq.18). The 
corresponding point candidates in model data present then the same surface type with similar curvedness as the requested points randomly selected in scene data.

$$
\begin{gathered}
\psi\left(p_{i}\right)=d_{f}<T h_{d f} \text { where } d_{f}=c_{p_{i}}-c_{q_{i}} \\
\text { for } L_{p_{i}}=L_{q_{i}}
\end{gathered}
$$

Moreover, data can be randomly sampled from specific surface type classes in scene data since a priori knowledge about the surface type in the overlapping section points is available. Although no priori knowledge is available, the idea to select points in specific surfaces type classes also reduces the chance to pick close points.

The $k$ random sampling can be set knowing the ratio $\zeta$ of the overlapping section points. Assuming $P^{*}$ the probability to have at least a set of three points in the overlapping section, $k$ can be determined using Eq.19.

$P^{*}=1-\left[1-(1-\zeta)^{3}\right]^{k} \Leftrightarrow k=\frac{\log \left(1-P^{*}\right)}{\log [1-(1-\zeta)]^{3}}$

\section{ICP variant}

Let us recall $P=\left\{p_{i}\right\}, i=1 \ldots m$ and $Q=\left\{q_{i}\right\}, i=1 \ldots n$, two data sets to align, which we call respectively the scene data (the moving set) and the model data (the fixed set) with generally $m \neq$ $n$. ICP algorithm aims to determine the optimal transformation, the rotation $R$ and the translation $T$, (producing the best alignment of $P$ and $Q$ so that $Q=R P+T$ ) from correspondences.

\section{1- Searching correspondences}

The matching step is traditionally based on the closest point criterion using Euclidean distance:

$$
\operatorname{Corres}(p)=\arg \min _{q \in Q} d(p, q) \leq T h_{1}
$$

where

$$
d(p, q)=d_{e}=\|p-q\|
$$

$T h_{1}$ is the threshold defining the accepted maximum distance.

However, a specific distance $d_{s}$ combining Euclidean distance $d_{e}$ with curvature distance $d_{c}$ defined from principal curvatures $\kappa_{1}$ and $\kappa_{2}$ is proposed here (Eqs.22 and 23) to take into account the curvature similarity besides the points position for the correspondences searching.

$$
d(p, q)=d_{s}=\lambda d_{e}+(1-\lambda) k d_{c}, k \in \mathbb{R}^{*+}
$$

Where,

$$
\begin{aligned}
& d_{c}=\sqrt{\left(\rho_{1 p}-\rho_{1 q}\right)^{2}+\left(\rho_{2 p}-\rho_{2 q}\right)^{2}}, \\
& \rho_{1}=\frac{1}{\kappa_{1}}, \rho_{2}=\frac{1}{\kappa_{2}}, \lambda \in[0,1]
\end{aligned}
$$


And $k$ is a constant to normalize $d_{c}$.

\section{2- Computation of $(R, T)$}

$(R, T)$ is classically computed by solving the optimization problem in least-squares sense where the associated P-P objective function is given in Eq.16. The associated P-Pl objective function $\mathrm{f}$ is provided in Eq.24. $\mathrm{N}_{\Omega}$ is the number of correspondences for both.

$$
f\left(R, T,\left(p_{i}, q_{i}\right)\right)=\sum_{i=1}^{N_{\Omega}}\left(\left(R p_{i}+T-q_{i}\right)^{T} n_{i}\right)^{2}
$$

The P-Pl method has been proved to be robust since the algorithm minimizes the sum of squared distances between the tangent plane at $\mathrm{q}_{\mathrm{i}}$ (estimated from its $\mathcal{H}$-neighbour points).

However, imprecisions can be induced especially when the neighbor points present high curvatures due to the least squares estimation of the tangent plane from these ones. Hence, the objective function has been improved by combining P-P and P-Pl approaches with automatic weights setting (Eq.25), to handle the classical P-Pl method limitation.

$$
(R, T)=\min _{R, T} \sum_{i=1}^{N_{\Omega}} w_{i}\left[\alpha_{i}\left\|R p_{i}+T-q_{i}\right\|^{2}+\left(\beta_{i}\left(R p_{i}+T-q_{i}\right)^{T} n_{i}\right)^{2}\right]
$$

$w_{i}$ are weights close to one indicating the reliable correspondences. They can be defined using Huber [30] or Tuckey criterion [31].

$\left(\alpha_{i}, \beta_{i}=1-\alpha_{i}\right)$ are weights to balance the contribution of P-P or P-P1 approaches in the optimization algorithm. They are automatically set according to the knowledge of the surface type at each point (Table II).

\section{3- Stopping criteria}

The algorithm stops as soon as one of the following conditions is satisfied:

- the Mean Squared Error (MSE) difference between two successive iterations is sufficiently small;

- the MSE is sufficiently small;

- the maximum allowed number of iterations has been reached.

\section{Experimental results}


A preliminary test of our algorithms on simulated data with additional generated Gaussian noises of $\pm 10 \mathrm{~nm}$ and $\pm 400 \mathrm{~nm}$ was achieved. The MSE (Mean Square Error)and the absolute RMS (RootMean-Square) and PV (Peak-to-Valley) variations are of sub-nanometer level whatever the coarse registration methods, which validates the robustness of our proposed approaches.

Therefore, the measurement of a cube standard is performed by a X-ray $\mu \mathrm{CT}$ system (X-ray microComputed Tomography [32]). The $\mu \mathrm{CT}$ Carl Zeiss METROTOM 800 is used here for the scanning of the manufactured material standard (Fig.4). It was specially achieved through the use of maintenance-free micro-focus X-ray tube technology. Extremely small focal points enable razorsharp projection images on the detector, leading to the foundation of a high measuring accuracy. The Carl Zeiss METROTOM $800 \mu \mathrm{CT}$ detector delivers almost three million pixels for very high detail recognition.

In this $\mu \mathrm{CT}$ system, the specimen can be located in any position in the beam path via a continuously adjustable travel mechanism. The detector is always optimally illuminated with the part projection. Together with the vertical adjustment, this function allows one to enlarge specific areas of the parts to measure details in relation to the entire specimen. Once the 2D projection images are acquired, specific software based on filtered back-projection was used to reconstruct the volume model (3D). More than one million points are recorded to cover the entire specimen surfaces. The registration of the $\mu \mathrm{CT}$ measurement data with the CAD data is carried out using the implemented methods.

The cube standard is made of aluminum with $15 \mathrm{~mm}$ of side length (Fig.5 (a)).

Afterward, the $\mathrm{CAD}$ data and $\mu \mathrm{CT}$ measurement have been aligned using the proposed curvaturebased registration methods. Its CAD and measurement data (Fig.5 (b)) are respectively composed of 587206 and 1913761 points.

The CAD surface type and curvedness maps are respectively provided in Fig.5 (c and d). The measurement surface type and curvedness maps on a local region are respectively illustrated in Fig.5 (e and f). Different surface types are distinctly identified depending on the meshing discretization, like the holes recognized as spherical cup (-4), the edge as ridge (2) and the cube flat side as plane (5), etc.

\section{1- Coarse registration}

The enhanced Hough and Ransac methods have been applied on points of a specific chosen surface type. This one should be selected according to its points distribution to avoid close points for Ransac approach while decreasing the counter of the wrong transformations for Hough method. Since the number of points of the same surface type is least, the algorithm complexity and the computational time are consequently reduced for a higher performance. However, a low number of 
points can lead to an unsatisfying coarse alignment due to noises. Therefore, a compromise should be found when selecting the surface type to be used for Ransac or for Hough methods. Here, Hough and Ransac have been applied on the surface type corresponding to $L=2$ (ridge). Thus, instead of a complexity of $587206 \times 1913761$ induced by basic Hough, our curvature based-Hough engenders at worst $10051 \times 430244$ local transformations calculation operations, which represents a reduction of $99.62 \%$.

For the Hough table, the threshold $\varepsilon$ in Eq.15 is split into two complementary thresholds, $\varepsilon_{\mathrm{R}}=$ $10^{-3}$ to delimit the rotation radian angles similarity and $\varepsilon_{\mathrm{T}}=1$ to delimit the translation components similarity. The maximum number of counter is 2018 which corresponds to $\mathrm{R}=[0.01$, $1.05,0.13]$ and $\mathrm{T}=[4.09,-8.27,-4.87]$ (Table III).

For Ransac, the k-random sampling set to 20 have been generated, for which the randomly selected points in $\mathrm{Q}$ are spaced within $2 \mathrm{~mm}$ at least. The two thresholds for the closest points criterion and for the accepted maximum value $\mathrm{n}$ are set to $0.01 \mathrm{~mm}$ and 1500 . These thresholds can be set considering the prior knowledge of the ratio of the considered surface type points present in the overlapping areas and the ratio of noisy points. The retained transformation is $\mathrm{R}=[-0.14,-1.12$, $0.14]$ and $\mathrm{T}=[3.93,-9.01,-4.32]$ (Table III).

A manual coarse alignment had been carried out primarily to supply a rough alignment reference for a rough comparison with our algorithm results (Table III). The quality of the obtained coarse alignments can be subsequently thorough and characterized from the fine registration results since they define the ICP initialization. As illustrated in Fig.6 (d) and reported in Table VII, the three methods converge to the identical order of MSE, RMS and PV for both classical fine registration algorithms (P-P and P-Pl) with different iterations. E.g.: for P-P, the obtained Manual MSE is of $2.1244 \times 10^{-4} \mathrm{~mm}$ after 16 iterations, while Hough and Ransac MSE are respectively $2.1138 \times 10^{-4}$ $\mathrm{mm}$ and $2.1134 \times 10^{-4} \mathrm{~mm}$ after 38 and 27 iterations. Higher is the quality of the rough alignment lower is the required number of iterations for convergence. The obtained Ransac coarse alignment quality is greater than Hough result with lesser computational time (720 s versus $2520 \mathrm{~s}$ ). However, Hough provides constant and repeatable results compared to Ransac which is based on a random sampling.

\section{2- Fine registration}

The proposed curvature-based fine registration including the combination of P-P and P-P1 minimization methods and the curvature distance used for the matching step, have been studied using the above three crude alignments. 
To consider the performance of P-P and P-P1 combination (denoted "Cb") minimization algorithm, the resulted MSE, RMS, PV are compared with the ones obtained using separately P-P and P-P1 algorithms. This analysis has been undertaken three times using a different rough alignment among Ransac, Hough and Manual results. For any crude alignment, the P-Pl confirms undeniably its advantage against the P-P (Tables IV, V and VI).

The combination approach $\mathrm{Cb}$ is firstly tested with a global set of weights applied on all point pairs, $\alpha_{\mathrm{i}}=\alpha$ and $\beta_{\mathrm{i}}=\beta$. $\alpha$ is varied from 0.1 to 0.9 since $\alpha=0$ corresponds to P-Pl and $\alpha=$ 1 to P-P (Tables IV, V and VI). The combination $\mathrm{Cb}$ provides advantageous results for $\alpha$ from 0.1 to 0.5 (i.e. by attributing much importance to P-P1), regardless of whatever the coarse alignment method. The minimum MSE and RMS values are reached for $\alpha=0.3$. The obtained MSE and RMS are of $3.9935 \times 10^{-5}$ and $1.9984 \times 10^{-3}$ for manual coarse (Table IV), $3.9944 \times 10^{-5}$ and $1.9986 \times 10^{-3}$ for Hough coarse (Table V) as well as $3.9544 \times 10^{-5}$ and $1.9909 \times 10^{-3}$ for Ransac coarse (Table VI).

Secondly for an automatic set of $\left(\alpha_{i}, \beta_{i}\right)$ different combinations of their values have been achieved and evaluated by mostly weighting with $\alpha_{i}=0.3, \beta_{i}=0.7$ according to the presented surface type while focusing on $[0.1,0.5] \alpha_{i}$ range.

The retained weights related to the automatic set (denoted AUTO) are reported in Table II with the lowest MSE and RMS values; E.g. $3.7555 \times 10^{-5}$ and $1.9379 \times 10^{-3}$ for Manual, $3.6614 \times 10^{-5}$ and $1.9147 \times 10^{-3}$ for Hough, $3.6768 \times 10^{-5}$ and $1.9193 \times 10^{-3}$ for Ransac (Tables IV, V and VI).

The influence of $\lambda$ parameter is investigated on MSE, PV and RMS issued of the three minimization approaches (P-P, P-Pl and Cb) and highlighted respectively in Fig.6 (e, f, g, h, i, j, k, 1 and m) for Manual, Hough and Ransac. Quite similar MSE behaviors obtained from P-P (Fig.6 (e)), from P-P1 (Fig.6 (f)) and from the combination of both (Fig.6 (g)) are noticed where the common minimum is reached generally at $\lambda=0.3$. The same observation is available for RMS curves. It reveals the effect of the curvature distance dc in ds and its advantage instead of using only Euclidean distance $d_{e}$. Comparison examples of MSE values: $1.9949 \times 10^{-3}(\lambda=0.3)$ versus $2.1244 \times 10^{-3}(\lambda=1)$ for P-P from manual (Fig.6 (e) and table IV), $5.2620 \times 10^{-4}$ versus $5.5097 \times 10^{-4}$ for P-P1 from Hough (Fig.6 (f) and Table V), $3.3964 \times 10^{-5}$ versus $3.6768 \times 10^{-5}$ for the Cb from Ransac (Fig.6 (g) and Table VI).

\section{3- Result analysis and discussion}

Through the previous analyses, the benefit of the proposed curvature based-fine algorithm was shown. The obtained MSEs are $3.4976 \times 10^{-5}, 3.4067 \times 10^{-5}$ and $3.3964 \times 10^{-5}$ for Manual, Hough and Ransac coarses respectively while $2.1244 \times 10^{-4}, 2.1138 \times 10^{-4}$ and $2.1134 \times 10^{-4}$ for P-P as well as $6.1326 \times 10^{-5}, 5.5097 \times 10^{-5}$ and $5.4155 \times 10^{-5}$ for P-Pl (Table VII). Regardless of the selected method, 
the PV, which combines intrinsic errors of the artefact and intrinsic errors of the CT system, is approximately of $20 \mu \mathrm{m}$.

The representative errors linked to the artefact are the form errors, the waviness and the roughness. Form errors and waviness are generated by the milling machine used for the manufacturing of the artefact. Thus, such kind of machine is equipped with numerous precision guiding systems presenting error motions of some micrometers, causing then form errors and waviness with similar level. The roughness depends on the selected milling parameters and tool and can be evaluated to few hundreds of nanometer.

The errors generated by CT can be distinguished into systematic and random errors. Systematic errors, or repeatable errors, are introduced by an inaccuracy inherent in the system, whereas random errors are caused by unknown and unpredictable changes in the system. Numerous errors sources in the CT can be identified such as: resolution of the source of the X-ray, resolution and linearity errors of the detector, positioning errors of the artefact in the working space, error motions and positioning errors of the spindle, positioning of the detector, reconstruction of the numerous collected 2D radiology, number of orientation of the artefact, etc.

Additionally, thermal drift is considered as a considerable source of error and can impact both the artefact and the CT system.

\section{Conclusion}

Three novel curvature-based registration methods including two coarse registration approaches and an ICP variant technique have been detailed in this paper. Hough and Ransac methods are traditionally used for coarse registration to cope with PCA limitations. Both methods have been enhanced by introducing curvature parameters exploitation in the algorithm for advanced performance. Indeed, the two algorithms are applied on specific areas selected and identified using surface type and curvedness similarity constraints, which considerably reduce the complexity and the computational time. These curvature parameters are preliminary calculated for each vertex of meshed data with a controlled meshing discretization. Local transformations computation endowed by Hough exhaustive search is only applied on points of the same surface type. The random sampling of points to be paired and their correspondences searching in Ransac based-darces algorithm are focused on a reduced area specified by the mixture of the surface type and curvedness similarity constraints. For fine registration, two contributions improving the matching step and the optimization problem definition have been carried out and proved to be performant compared to classical algorithms. The reliability of correspondences searching is based on the use of specific distance involving the combination of Euclidean distance and curvature distance. Curvature 
distance is defined from principal curvatures to measure the invariant curvature features similarity, which importance is weighted by $\lambda$ close to zero. The second contribution consists of combining the P-P minimization with P-P1 minimization, which both are automatically weighted depending on the pre-calculated surface type of the considered paired points. Different applications have been handled to validate the proposed algorithms. Through these applications, the observed performance induced by curvature parameters introduction in Hough and in Ransac have been highlighted while the influence of the curvature similarity measure employed for fine registration as well as the benefit of the novel objective function, have been emphasized. This one has been revealed to be robust to noises instead of adopting separately P-P and P-Pl minimization approach.

\section{Acknowledgments}

The authors sincerely thank the EMPIR organization. The EMPIR is jointly funded by the EMPIR participating countries within EURAMET and the European Union (15SIB01: FreeFORM).

The authors are grateful to Dr. Frank Thibault from Carl Zeiss in France for the CT measurements on our artefacts. 


\section{References}

[1] A. Weckenmann, X. Jiang, K. D. Sommer, U. Neuschaefer-Rube, J. Seewig, L. Shaw, T. Estler, Multisensor data fusion in dimensional metrology, CIRP AnnalsManufacturing Technology 58 (2009) 701-721.

[2] L. Shaw, A. Weckenmann, Automatic registration method for hybrid optical coordinate measuring technology, CIRP Annals-Manufacturing Technology 60 (2011) 539-542.

[3] Díez, Y., Roure, F., Lladó, X., \& Salvi, J. (2015). A qualitative review on 3d coarse registration methods. ACM Computing Surveys (CSUR), 47(3), 45.

[4] Z. Zhang, C. Pan and S. Ma, An automatic method of coarse registration between multi-source satellite images, Proceedings of IEEE Conference on Intelligent Sensors, Sensors Network and Information (2004) 205-209.

[5] A. Myronenko, X. Song, Point set registration: Coherent point drift, IEEE Transactions on Pattern Analysis and Machine Intelligence 32 (2010) 2262-2275.

[6] M. Attene, B. Falcidieno, M. Spagnuolo, Hierarchical mesh segmentation based on fitting primitives, The Visual Computer 22 (2006) 181-193.

[7] A. Johnson, Q-S. Kang, Y-L. Yang, S-M. Hu, Registration and Integration of Textured 3-D Data, Proc. 3DIM (1997).

[8] H. Pottmann, Q. Huang, Y. Yang, Geometry and convergence analysis of algorithms for registration of 3-D shapes, International Journal of Computer Vision 67 (2006) 277-296.

[9] W-L. Li, Z-P. Yin, Y-A. Huang and Y-L. Xiong, Automatic registration for 3D shapes using hybrid dimensionality-reduction shape descriptions, Pattern Recognition 44 (2011) 2926-2943.

[10] R. Rantoson, C. Stolz, D. Fofi, F. Meriaudeau, Non contact 3D measurement scheme for transparent objects using UV structured light, $20^{\text {th }}$ IEEE International Conference on Pattern Recognition (ICPR) (2010) 1646-1649.

[11] G. Xiao, S.H. Ong, K.W.C. Foong, Registration of partially overlapping surfaces by rejection of false point correspondences, Pattern Recognition 39 (2006) 373-383.

[12] S.M. Yamany, A.A. Farag, Surfacing signatures: An orientation independent freeform surface representation scheme for the purpose of objects registration and matching, IEEE Transactions on Pattern Analysis and Machine Intelligence 24 (2002) 1105-1120. 
[13] H. Zhao, N. Anwer, P. Bourdet, Curvature-based registration and segmentation for multisensor coordinate metrology, Procedia CIRP 10 (2013) 112-118

[14] J. Illingworth, J. Kittler, The adaptive hough transform, IEEE Transactions on Pattern Analysis and Machine Intelligence 9 (1987) 689-698.

[15] C-S. Chen, Y-P. Hung, J-B. Cheng, Ransac-based darces: A new approach to fast automatic registration of partially overlapping range images, IEEE Transactions on Pattern Analysis and Machine Intelligence 21 (1999) 1229-1234.

[16] P. Besl, N. McKay, A method for registration of 3-D shapes, IEEE Transactions on Pattern Analysis and Machine Intelligence 14 (1992) 239 - 256.

[17] Y. Chen, G. Medioni, Object modeling by registration of multiple range images, IEEE International Conference on Robotics and Automation (1991) 2724 2729 .

[18] S. Rusinkiewicz, M. Levoy, Efficient variants of the ICP algorithm, IEEE Third International Conference on 3-D Digital Imaging and Modeling (2001) 145-152.

[19] M. Greenspan, M. Yurick, Approximate k-d tree search for efficient ICP, IEEE Fourth International Conference on 3-D Digital Imaging and Modeling (2003) 442448.

[20] R. Benjemma, F. Schmitt, Fast global registration of 3D sampled surface using a multi-z-buffer technique, Journal of Imaging and Vision Computing 17 (1999) 113123.

[21] G.K.L. Tam, Z-Q. Cheng, Y-K. Lai, F.C. Langbein, Y. Liu, D. Marshall, R.R. Martin, X-F. Sun, P. L. Rosin, Registration of 3D Point Clouds and Meshes: A Survey from Rigid to Nonrigid Visualization and Computer Graphics, IEEE Transactions on Visualization and Computer Graphics 19 (2013) 1199-1217.

[22] Y. Yang, S.H. Ong, K.W.C. Foong, A robust global and local mixture distance based non-rigid point set registration, Pattern Recognition 48 (2015) 156-173.

[23] T. Masuda, N. Yokoya, A robust method for registration and segmentation of multiple range images, Computer Vision and Image Understanding 61 (1995) 295307.

[24] D. Chetverikov, D. Svirko, D. Stepanov, P. Krsek, The trimmed iterative closest point algorithm, IEEE $16^{\text {th }}$ International Conference in Pattern Recognition 3 (2002) $545-548$.

[25] A. Lorusso, D.W. Eggert, R.B. Fisher, A comparison of four algorithms for 
estimating 3-D rigid transformations, British Conference on Machine Vision 1 (1995) 237-246.

[26] D. Cohen-Steiner, J.M. Morvan, Restricted Delaunay Triangulation and Normal Cycle, Proceedings of $19^{\text {th }}$ Annual ACM Symposium on Computational Geometry (2003) 312-321.

[27] M. Meyer, M. Desbrun, P. Schroder, A.H. Barr, Discrete differential-geometry operators for triangulated 2-manifolds, Proceedings of Visualization and Mathematics (2002) 35-57.

[28] D. Meek, D. Walton, On surface normal and Gaussian curvature estimations given data sampled from a smooth surface, Journal of Computer Aided Geometric Design 17 (2000) 521-543.

[29] J.J. Koenderink, R. J. Van Doorn, Surface shape and curvature scales, Journal of Imaging and Vision Computing 10 (1992) 557-565.

[30] P.J. Huber, Robust statistics, Springer Berlin Heidelberg (2011).

[31] A.C. Öztireli, G. Guennebaud, M. Gross, Feature Preserving Point Set Surfaces based on Non-Linear Kernel Regression, Computer Graphics Forum 28 (2009) 493-501.

[32] J. P. Kruth, M. Bartscher, S. Carmignato, R. Schmitt, L. De Chiffre, A. Weckenmann Computed tomography for dimensional metrology, CIRP Annals - Manufacturing Technology 60 (2011) 821-842. 


\section{Figure captions}

Fig. 1: (a) Normal curvature illustration, (b) Voronoï-based cell generation for the local region B definition, (c) Dihedral angle illustration, (d) Illustration of $\boldsymbol{n p}$ calculation where $\boldsymbol{\varkappa}=\mathbf{6}$. ---- 21

Fig. 2: Hough algorithm illustrations: (a) The basic; (b) The enhanced.------------------------------ 22

Fig. 3: Ransac-based darces registration constraints illustrations related to the Step 2: (a) $\mathrm{q}_{2}$

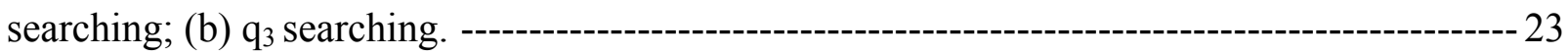

Fig. 4: Cube illustrations: (a) Its photo; (b) Its measurement data; (c) Its CAD surface type map; (d) Its CAD curvedness map; (e) and (f) Zoom of its measurement surface type and curvedness maps

Fig. 5: Results illustrations of the CAD and its measurement registration: (a) Initial alignment; (b) Coarse registration; (c) Fine registration; (d) MSE Curves obtained using the curvature-based registration with different coarse registration methods; (e) (f) (g) Final MSE values obtained with different values of $\lambda$ using respectively P-P, P-Pl minimization approach and the combination of both (Cb). (h) (i) (j) Finale PV values obtained with different values of $\lambda$ using respectively P-P, P-P1 minimization approach and the combination of both $(\mathrm{Cb})$. (k) (1) (m) Final RMS values obtained with different values of $\lambda$ using respectively P-P, P-Pl minimization approach and the combination of both $(\mathrm{Cb})$. Note that M: Manual, H: Hough and R: Ransac. 26 


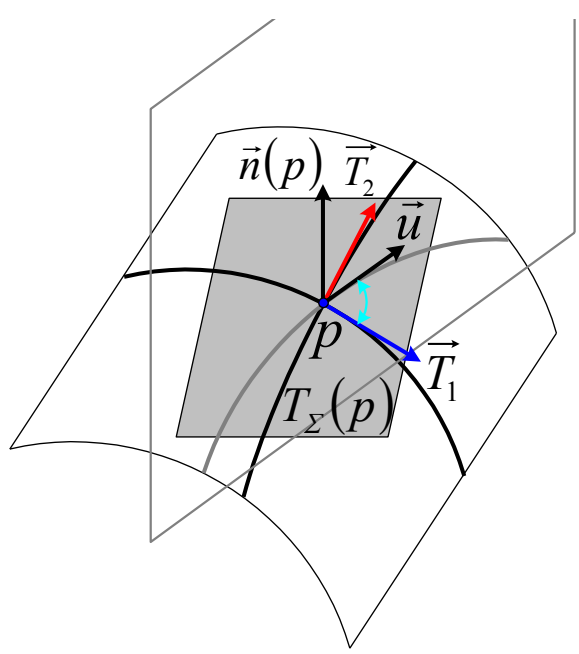

(a)

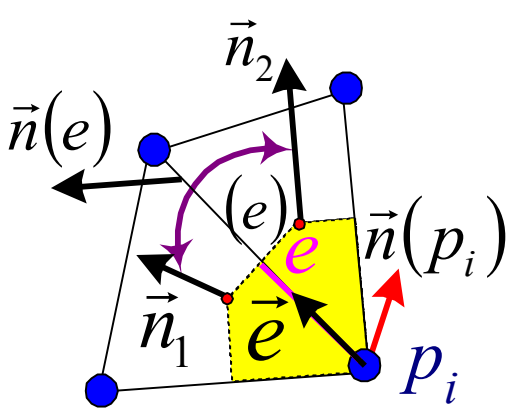

(c)

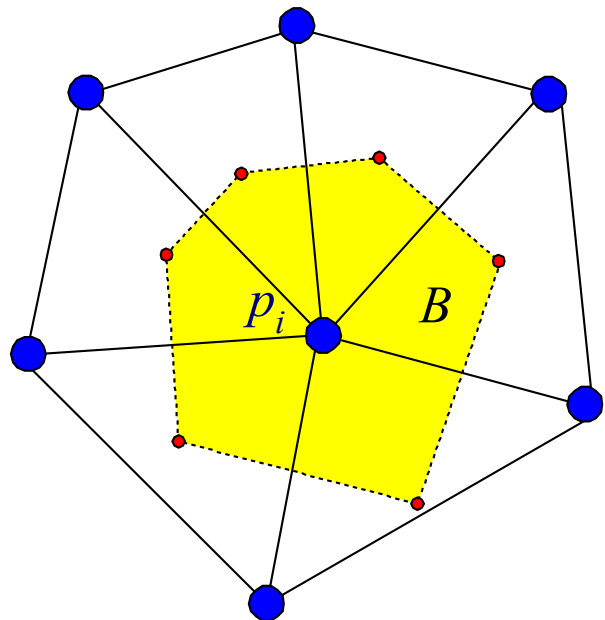

(b)

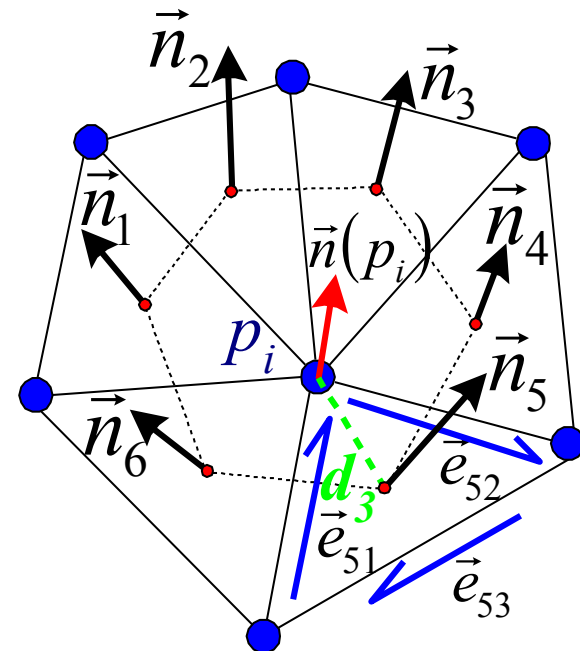

(d)

Fig. 1: (a) Normal curvature illustration, (b) Voronoï-based cell generation for the local region $B$ definition, (c) Dihedral angle illustration, (d) Illustration of $\overrightarrow{\boldsymbol{n}}(\boldsymbol{p})$ calculation where $\boldsymbol{\varkappa}=\mathbf{6}$. 


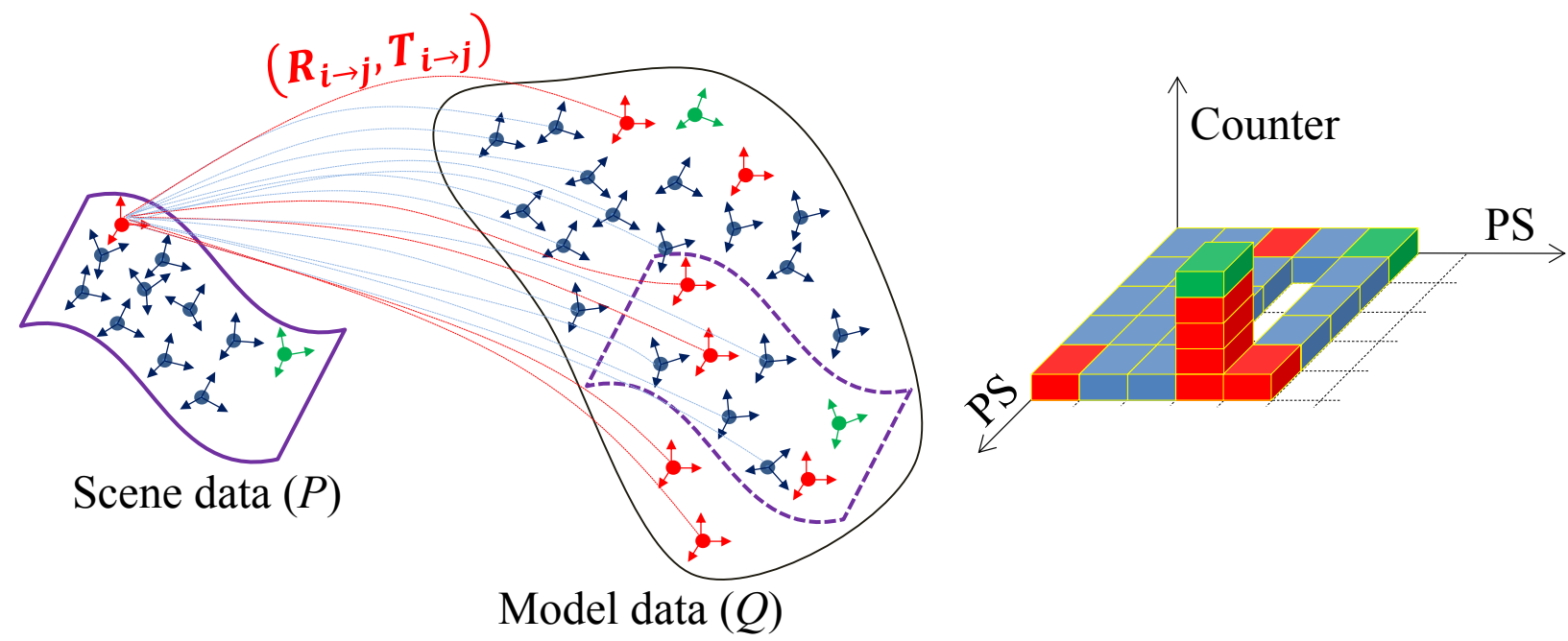

(a)

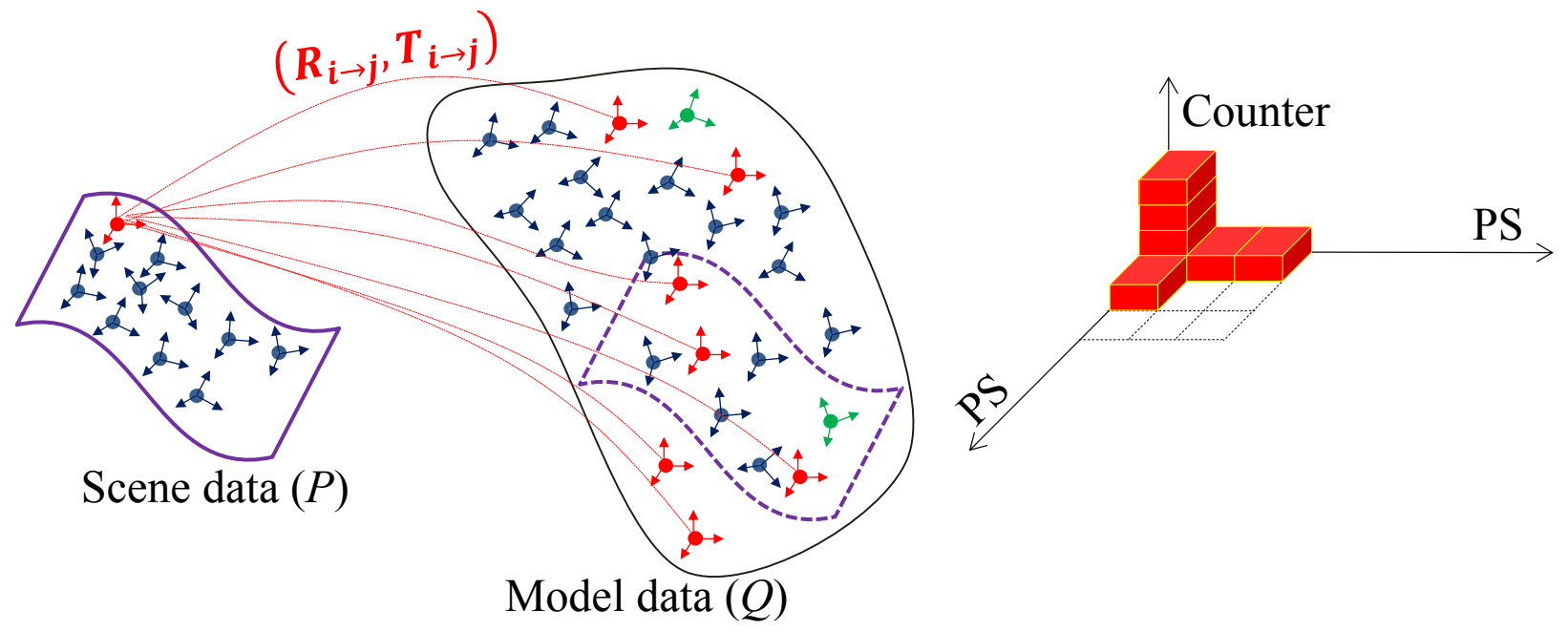

(b)

Fig. 2: Hough algorithm illustrations: (a) The basic; (b) The enhanced. 

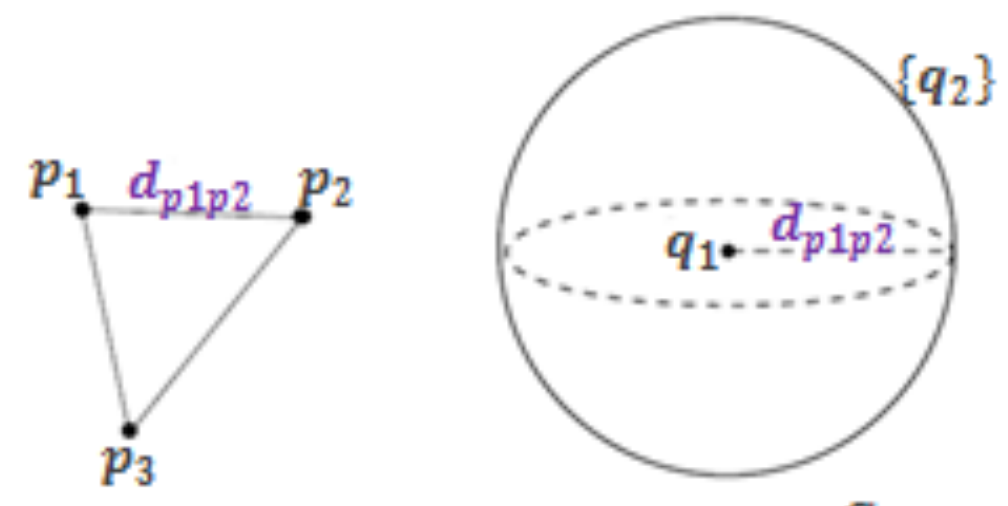

(a)

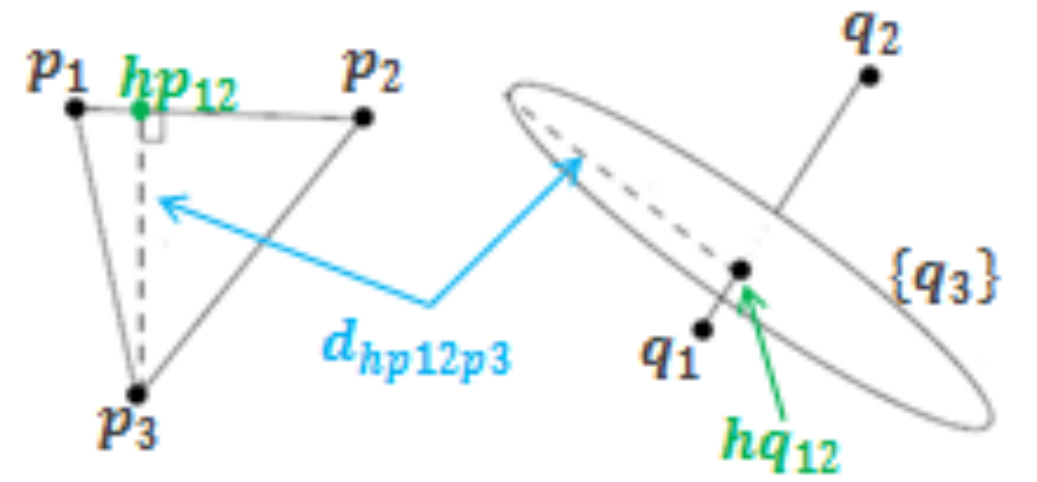

(b)

Fig. 3: Ransac-based darces registration constraints illustrations related to the Step 2: (a) $q_{2}$ searching; (b) $q_{3}$ searching. 


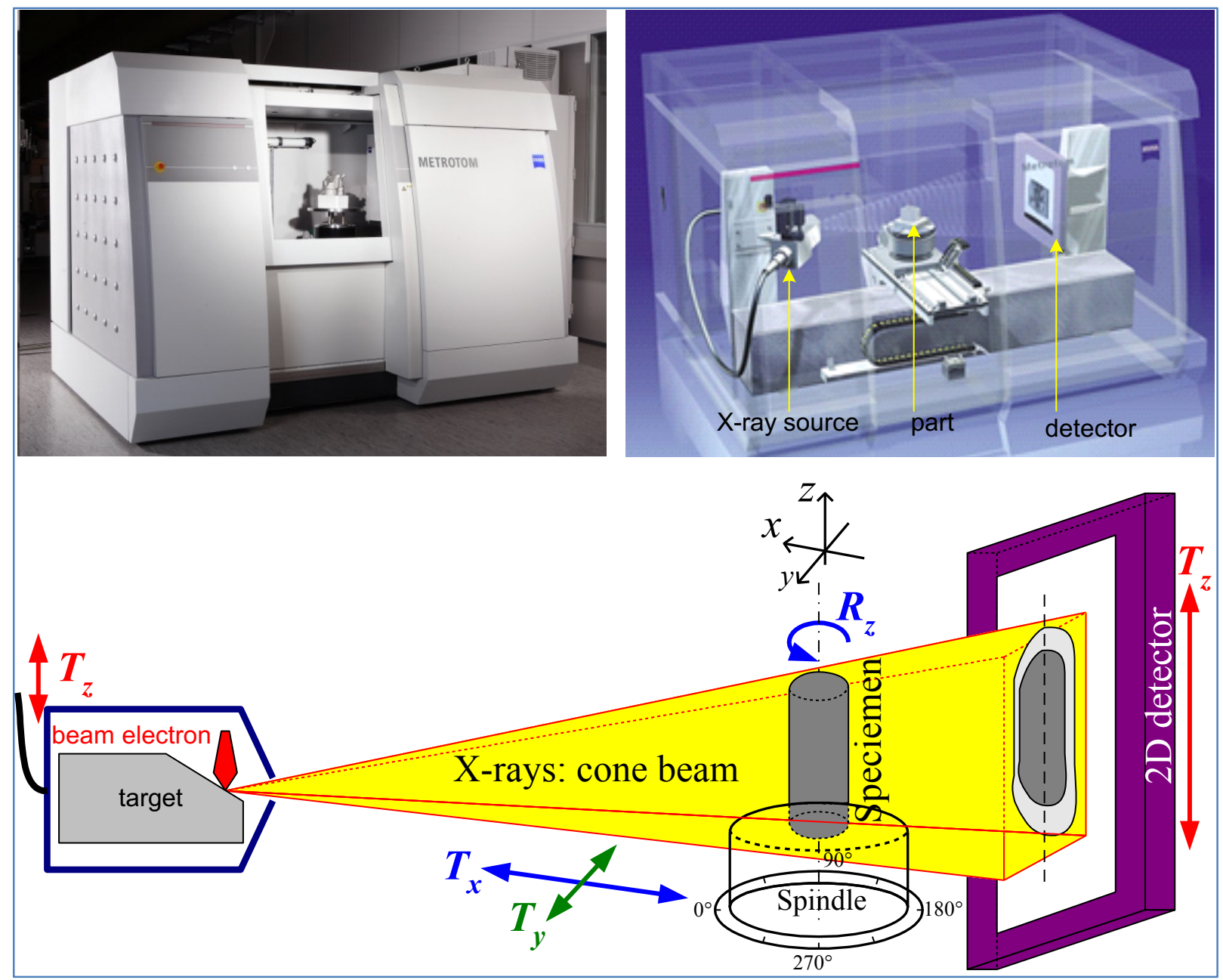

Fig. 4: Schema of the $\mu \mathrm{CT}$ equipped with $2 \mathrm{D}$ flat panel detector with cone beam (Carl Zeiss METROTOM 800CT). 


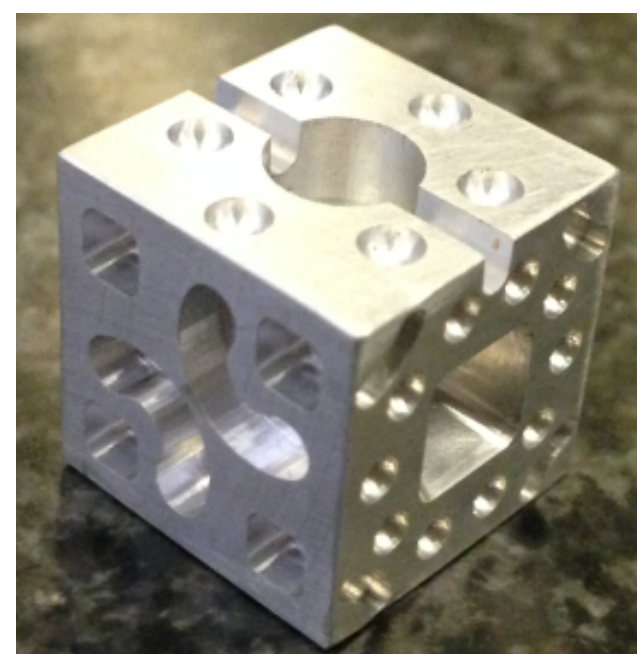

(a)

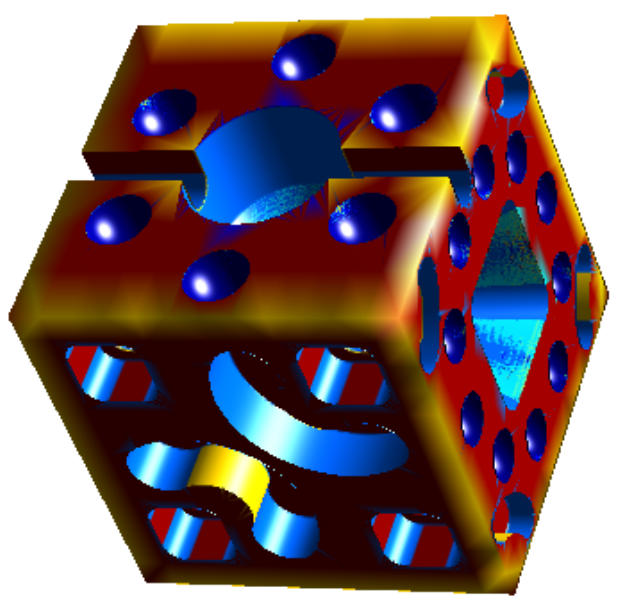

(c)

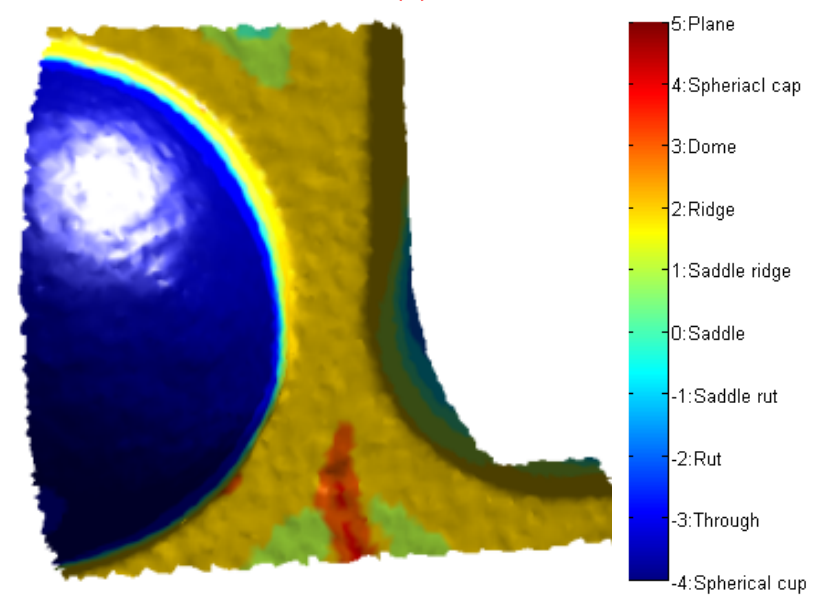

(e)

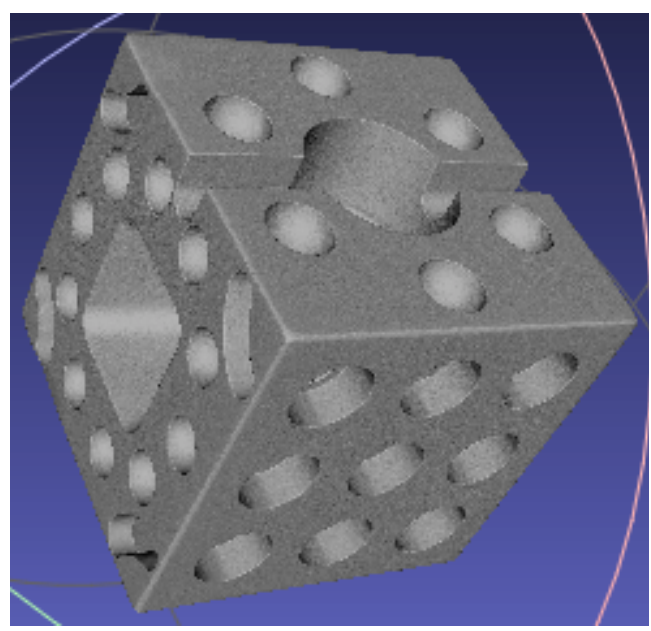

(b)
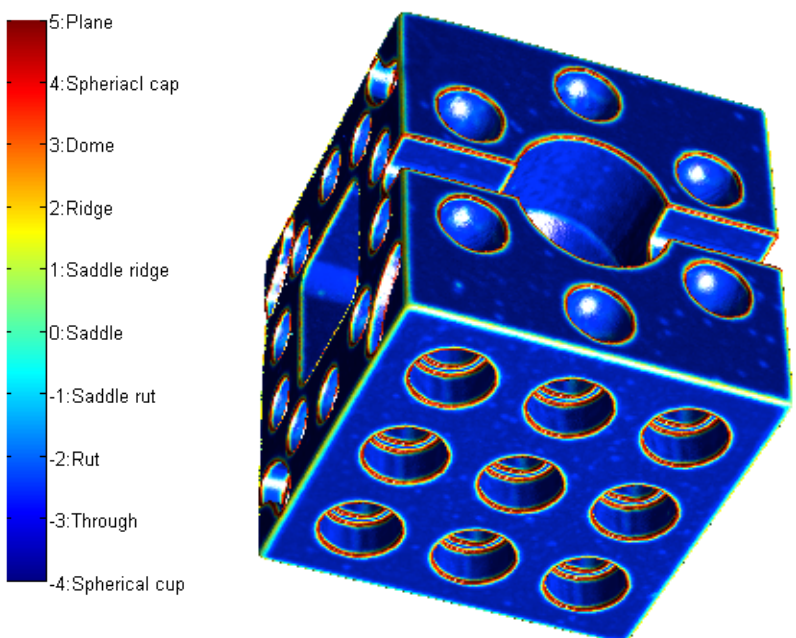

(d)

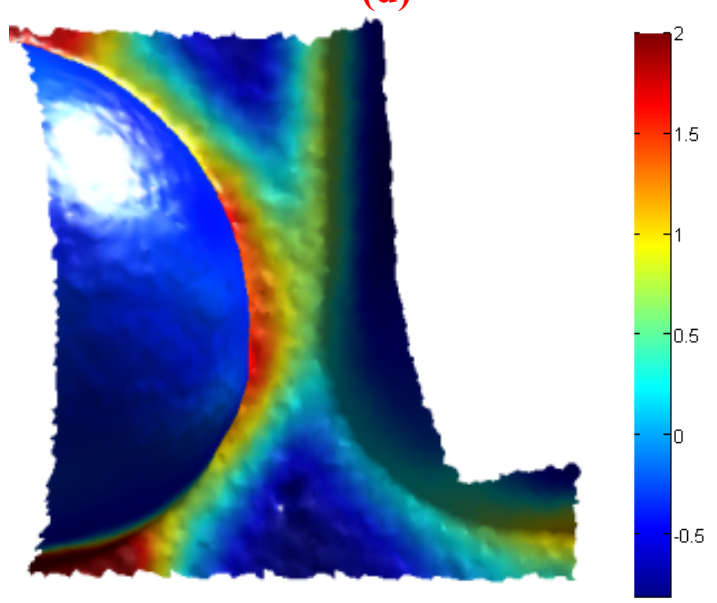

(f)

Fig. 5: Cube illustrations: (a) Its photo; (b) Its measurement data; (c) Its CAD surface type map; (d) Its CAD curvedness map; (e) and (f) Zoom of its measurement surface type and curvedness maps. 


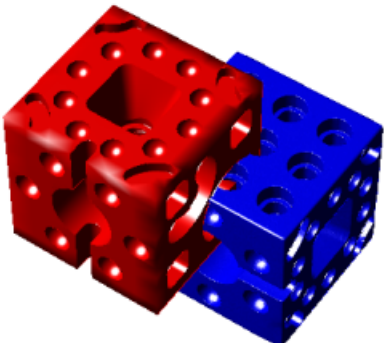

(a)

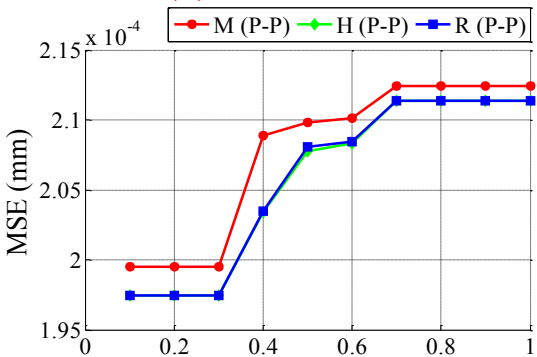

(e)

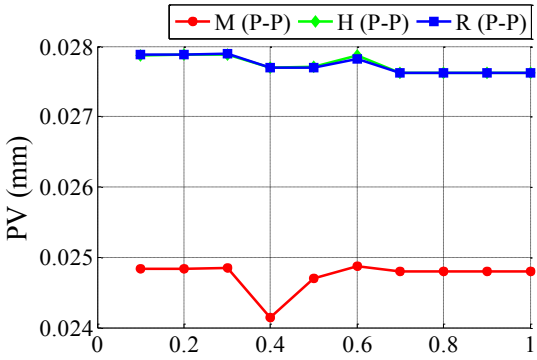

(h)

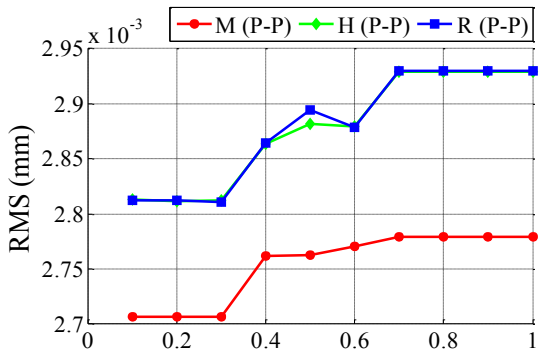

(k)

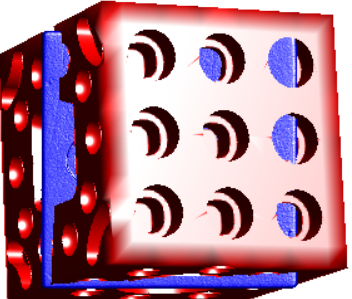

(b)

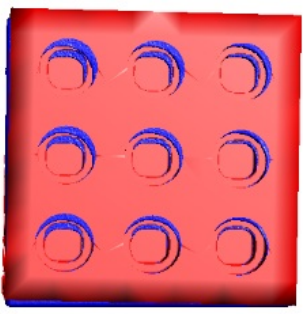

(c)

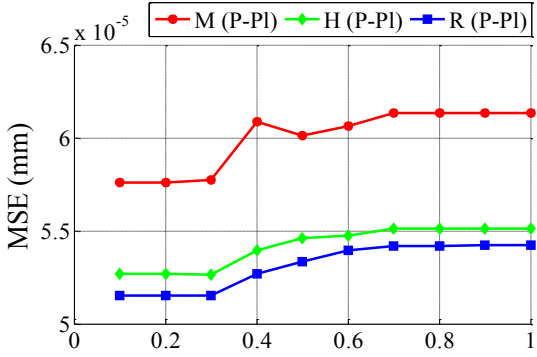

(f)

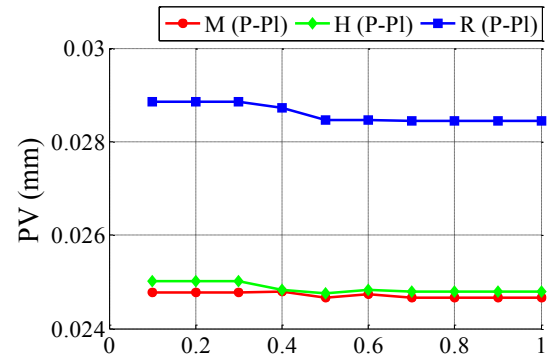

(i)

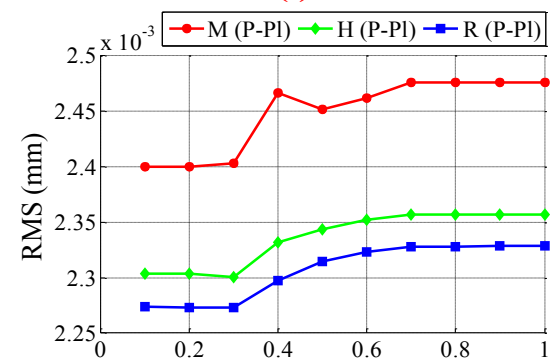

(I)

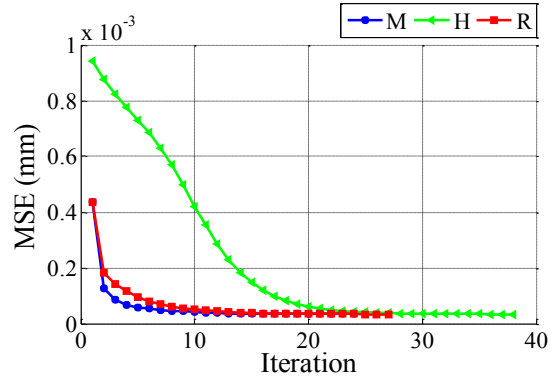

(d)

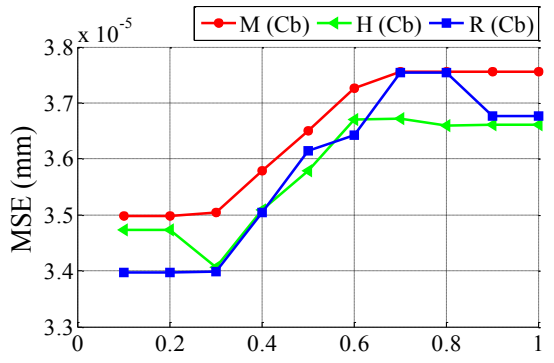

(g)

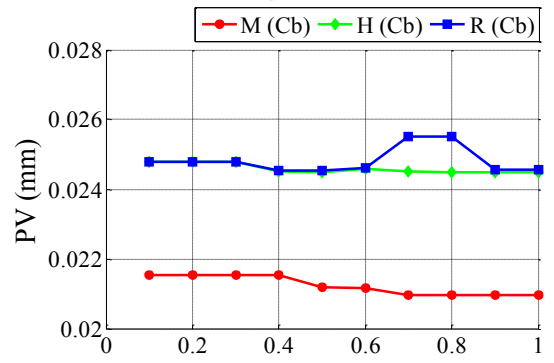

(j)

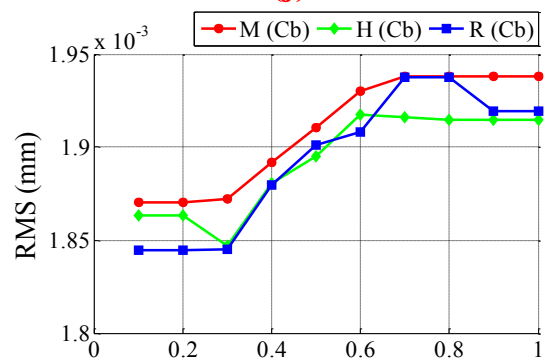

(m)

Fig. 6: Results illustrations of the CAD and its measurement registration: (a) Initial alignment; (b) Coarse registration; (c) Fine registration; (d) MSE Curves obtained using the curvature-based registration with different coarse registration methods; (e) (f) (g) Final MSE values obtained with different values of $\lambda$ using respectively P-P, P-P1 minimization approach and the combination of both (Cb). (h) (i) (j) Finale PV values obtained with different values of $\lambda$ using respectively P-P, PPl minimization approach and the combination of both (Cb). (k) (l) (m) Final RMS values obtained with different values of $\lambda$ using respectively P-P, P-Pl minimization approach and the combination of both (Cb). Note that M: Manual, H: Hough and R: Ransac. 


\section{Table captions}

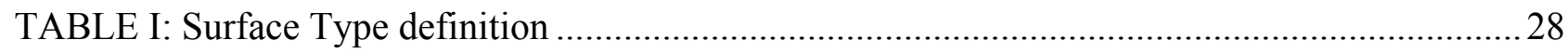

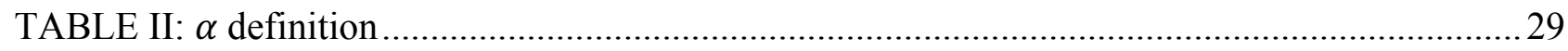

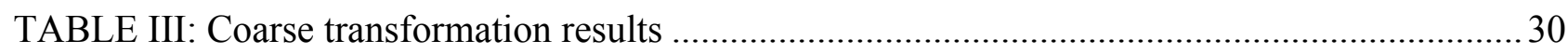

TABLE IV: P-P, P-Pl and Cb minimization tests from manual coarse .......................................... 31

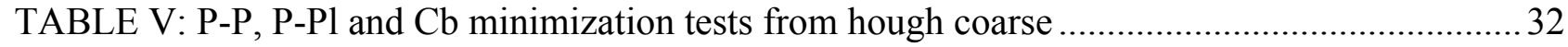

TABLE VI: P-P, P-Pl and Cb minimization tests from Ransac coarse ..........................................33

TABLE VII: Comparison of $\mathrm{Cb}$ with classical ICP using different coarse .....................................34 
TABLE I: SURfACE TYPE DEFINITION

\begin{tabular}{|c|c|c|c|c|c|c|c|}
\hline $\begin{array}{l}\text { Surface } \\
\text { type }\end{array}$ & Shape index $s$ & $L$ & Color & $\begin{array}{l}\text { Surface } \\
\text { type }\end{array}$ & Shape index $s$ & $L$ & Color \\
\hline $\begin{array}{l}\text { Spherical } \\
\text { cup }\end{array}$ & $s \in\left[-1,-\frac{7}{8}\right]$ & -4 & & $\begin{array}{l}\text { Spherical } \\
\text { cap }\end{array}$ & $s \in\left[1, \frac{7}{8}\right]$ & 4 & \\
\hline Through & $\left.s \in]-\frac{7}{8},-\frac{5}{8}\right]$ & -3 & & Dome & $s \in\left[\frac{5}{8}, \frac{7}{8}\right]$ & 3 & \\
\hline Rut & $\left.s \in]-\frac{5}{8},-\frac{3}{8}\right]$ & -2 & & Ridge & $s \in\left[\frac{3}{8}, \frac{5}{8}\right]$ & 2 & \\
\hline $\begin{array}{l}\text { Saddle } \\
\text { rut }\end{array}$ & $\left.s \in]-\frac{3}{8},-\frac{1}{8}\right]$ & -1 & & $\begin{array}{l}\text { Saddle } \\
\text { ridge }\end{array}$ & $s \in\left[\frac{1}{8}, \frac{3}{8}\right]$ & 1 & \\
\hline saddle & $\left.s \in]-\frac{1}{8}, \frac{1}{8}\right]$ & 0 & & Plane & $s=2$ & 5 & \\
\hline
\end{tabular}


TABLE II: $\alpha$ definition

\begin{tabular}{ccccccccccc}
\hline \hline$L$ & -4 & -3 & -2 & -1 & 0 & 1 & 2 & 3 & 4 & 5 \\
\hline$\alpha$ & 0.5 & 0.4 & 0.2 & 0.3 & 0.3 & 0.3 & 0.2 & 0.4 & 0.5 & 0 \\
\hline \hline
\end{tabular}


TABLE III: Coarse transformation results

\begin{tabular}{ccc}
\hline \hline \multicolumn{1}{c}{ Manual } & Hough & Ransac \\
\hline$R=[-0.15,-1.53,-0.21]$ & $R=[0.01 ;-1.05 ; 0.13]$ & $R=[-0.14 ;-1.12 ; 0.14]$ \\
$T=[4.82 ;-6.81 ;-7.86]$ & $T=[4.09 ;-8.27 ;-4.87]$ & $T=[3.93-9.01-4.32]$ \\
\hline
\end{tabular}


TABLE IV: P-P, P-Pl and $\mathrm{Cb}$ minimization tests from manual coarse

\begin{tabular}{|c|c|c|c|c|}
\hline \multicolumn{2}{|c|}{ Approach } & $\overline{P \text { PV (mm) }}$ & RMS (mm) & MSE (mm) \\
\hline \multicolumn{2}{|l|}{ P-P } & $2.4796 \times 10^{-2}$ & $2.7792 \times 10^{-3}$ & $2.1244 \times 10^{-4}$ \\
\hline \multicolumn{2}{|l|}{ P-PI } & $2.4664 \times 10^{-2}$ & $2.4764 \times 10^{-3}$ & $6.1326 \times 10^{-5}$ \\
\hline \multirow{9}{*}{$\begin{array}{l}0 \\
0 \\
0 \\
0\end{array}$} & $0.1-0.9$ & $2.3814 \times 10^{-2}$ & $2.2593 \times 10^{-3}$ & $5.1044 \times 10^{-5}$ \\
\hline & $0.2-0.8$ & $1.9454 \times 10^{-2}$ & $2.0450 \times 10^{-3}$ & $4.1820 \times 10^{-5}$ \\
\hline & $0.3-0.7$ & $2.0383 \times 10^{-2}$ & $1.9984 \times 10^{-3}$ & $3.9935 \times 10^{-5}$ \\
\hline & $0.4-0.6$ & $1.9705 \times 10^{-2}$ & $2.0399 \times 10^{-3}$ & $4.1449 \times 10^{-5}$ \\
\hline & $0.5-0.5$ & $2.3021 \times 10^{-2}$ & $2.2654 \times 10^{-3}$ & $5.1231 \times 10^{-5}$ \\
\hline & $0.6-0.4$ & $2.3456 \times 10^{-2}$ & $2.4081 \times 10^{-3}$ & $5.7759 \times 10^{-5}$ \\
\hline & $0.7-0.3$ & $2.3493 \times 10^{-2}$ & $2.4916 \times 10^{-3}$ & $6.1793 \times 10^{-5}$ \\
\hline & $0.8-0.2$ & $2.3704 \times 10^{-2}$ & $2.6157 \times 10^{-3}$ & $6.8002 \times 10^{-5}$ \\
\hline & $0.9-0.1$ & $2.3867 \times 10^{-2}$ & $2.7133 \times 10^{-3}$ & $7.3621 \times 10^{-5}$ \\
\hline AUTO & & $2.0965 \times 10^{-2}$ & $1.9379 \times 10^{-3}$ & $3.7555 \times 10^{-5}$ \\
\hline
\end{tabular}


TABLE V: P-P, P-Pl and $\mathrm{Cb}$ minimization tests from hough coarse

\begin{tabular}{|c|c|c|c|c|}
\hline \multicolumn{2}{|c|}{ Approach } & PV (mm) & RMS (mm) & MSE (mm) \\
\hline \multicolumn{2}{|l|}{ P-P } & $2.7624 \times 10^{-2}$ & $2.9289 \times 10^{-3}$ & $2.1138 \times 10^{-4}$ \\
\hline \multicolumn{2}{|l|}{ P-PI } & $2.4794 \times 10^{-2}$ & $2.3572 \times 10^{-3}$ & $5.5097 \times 10^{-5}$ \\
\hline \multirow{9}{*}{$\underbrace{0}_{0}$} & $0.1-0.9$ & $2.5033 \times 10^{-2}$ & $2.1498 \times 10^{-3}$ & $4.6216 \times 10^{-5}$ \\
\hline & $0.2-0.8$ & $2.5160 \times 10^{-2}$ & $2.0197 \times 10^{-3}$ & $4.0679 \times 10^{-5}$ \\
\hline & $0.3-0.7$ & $2.3974 \times 10^{-2}$ & $1.9986 \times 10^{-3}$ & $3.9944 \times 10^{-5}$ \\
\hline & $0.4-0.6$ & $2.3402 \times 10^{-2}$ & $2.0962 \times 10^{-3}$ & $4.3803 \times 10^{-5}$ \\
\hline & $0.5-0.5$ & $2.3737 \times 10^{-2}$ & $2.2226 \times 10^{-3}$ & $4.9401 \times 10^{-5}$ \\
\hline & $0.6-0.4$ & $2.7690 \times 10^{-2}$ & $2.3520 \times 10^{-3}$ & $5.5240 \times 10^{-5}$ \\
\hline & $0.7-0.3$ & $2.6914 \times 10^{-2}$ & $2.4726 \times 10^{-3}$ & $6.1138 \times 10^{-5}$ \\
\hline & $0.8-0.2$ & $2.7099 \times 10^{-2}$ & $2.6358 \times 10^{-3}$ & $6.9473 \times 10^{-5}$ \\
\hline & $0.9-0.1$ & $2.7760 \times 10^{-2}$ & $2.7784 \times 10^{-3}$ & $7.7192 \times 10^{-5}$ \\
\hline \multicolumn{2}{|l|}{ AUTO } & $2.4486 \times 10^{-2}$ & $1.9147 \times 10^{-3}$ & $3.6614 \times 10^{-5}$ \\
\hline
\end{tabular}


TABLE VI: P-P, P-Pl and Cb minimization tests from Ransac coarse

\begin{tabular}{|c|c|c|c|c|}
\hline \multicolumn{2}{|c|}{ Approach } & PV (mm) & RMS (mm) & MSE (mm) \\
\hline \multicolumn{2}{|l|}{$\mathbf{P}-\mathbf{P}$} & $2.7625 \times 10^{-2}$ & $2.9302 \times 10^{-3}$ & $2.1134 \times 10^{-4}$ \\
\hline \multicolumn{2}{|l|}{ P-PI } & $2.8438 \times 10^{-2}$ & $2.3288 \times 10^{-3}$ & $5.4234 \times 10^{-5}$ \\
\hline \multirow{9}{*}{$\underbrace{00}_{0}$} & $0.1-0.9$ & $2.5193 \times 10^{-2}$ & $2.1281 \times 10^{-3}$ & $4.5143 \times 10^{-5}$ \\
\hline & $0.2-0.8$ & $2.5228 \times 10^{-2}$ & $2.0091 \times 10^{-3}$ & $4.0165 \times 10^{-5}$ \\
\hline & $0.3-0.7$ & $2.4025 \times 10^{-2}$ & $1.9909 \times 10^{-3}$ & $3.9544 \times 10^{-5}$ \\
\hline & $0.4-0.6$ & $2.4330 \times 10^{-2}$ & $2.0957 \times 10^{-3}$ & $4.3918 \times 10^{-5}$ \\
\hline & $0.5-0.5$ & $2.3751 \times 10^{-2}$ & $2.2187 \times 10^{-3}$ & $4.9224 \times 10^{-5}$ \\
\hline & $0.6-0.4$ & $2.7702 \times 10^{-2}$ & $2.3482 \times 10^{-3}$ & $5.5142 \times 10^{-5}$ \\
\hline & $0.7-0.3$ & $2.6645 \times 10^{-2}$ & $2.5261 \times 10^{-3}$ & $6.3814 \times 10^{-5}$ \\
\hline & $0.8-0.2$ & $2.7106 \times 10^{-2}$ & $2.6351 \times 10^{-3}$ & $6.9436 \times 10^{-5}$ \\
\hline & $0.9-0.1$ & $2.7758 \times 10^{-2}$ & $2.7774 \times 10^{-3}$ & $7.7141 \times 10^{-5}$ \\
\hline \multicolumn{2}{|l|}{ AUTO } & $2.4551 \times 10^{-2}$ & $1.9193 \times 10^{-3}$ & $3.6768 \times 10^{-5}$ \\
\hline
\end{tabular}


TABLE VII: Comparison of $\mathrm{Cb}$ with classical ICP using different coarse

\begin{tabular}{lllll}
\hline \hline Approach & \multicolumn{1}{c}{ P-P } & P-PI & Cb. AUTO \\
\cline { 2 - 5 } & Unit $(\mathrm{mm})$ & $d_{g}(\lambda=1)$ & $d_{g}(\lambda=1)$ & $d_{g}(\lambda=0.2)$ \\
\hline \multirow{3}{*}{ Manual } & PV & $2.4796 \times 10^{-2}$ & $2.4664 \times 10^{-2}$ & $2.1528 \times 10^{-2}$ \\
\cline { 2 - 5 } & RMS & $2.7792 \times 10^{-3}$ & $2.4764 \times 10^{-3}$ & $1.8702 \times 10^{-3}$ \\
\cline { 2 - 5 } & MSE & $2.1244 \times 10^{-4}$ & $6.1326 \times 10^{-5}$ & $3.4976 \times 10^{-5}$ \\
\hline \multirow{3}{*}{ Hough } & PV & $2.7624 \times 10^{-2}$ & $2.4794 \times 10^{-2}$ & $2.4776 \times 10^{-2}$ \\
\cline { 2 - 5 } & RMS & $2.9289 \times 10^{-3}$ & $2.3572 \times 10^{-3}$ & $1.8471 \times 10^{-3}$ \\
\cline { 2 - 5 } & MSE & $2.1138 \times 10^{-4}$ & $5.5097 \times 10^{-5}$ & $3.4067 \times 10^{-5}$ \\
\hline \multirow{2}{*}{ Ransac } & PV & $2.7625 \times 10^{-2}$ & $2.8453 \times 10^{-2}$ & $2.4785 \times 10^{-2}$ \\
\cline { 2 - 5 } & RMS & $2.9302 \times 10^{-3}$ & $2.3301 \times 10^{-3}$ & $1.8447 \times 10^{-3}$ \\
\cline { 2 - 5 } & MSE & $2.1134 \times 10^{-4}$ & $5.4155 \times 10^{-5}$ & $3.3964 \times 10^{-5}$ \\
\hline \hline
\end{tabular}

